D) Check for updates

Cite this: Nanoscale, 2021, 13, 1016

Received 19th September 2020 Accepted 2nd December 2020

DOI: $10.1039 /$ d0nr06747d

rsc.li/nanoscale

\section{Revisiting the cellular toxicity of benzo[a]pyrene from the view of nanoclusters: size- and nanoplastic adsorption-dependent bioavailability $\dagger$}

\author{
Yunxia Ji, $\ddagger^{\mathrm{a}, \mathrm{b}}$ Yunqing Wang, $\hbar^{\mathrm{b}, \mathrm{c}}$ Dazhong Shen, (D) ${ }^{\mathrm{a}}$ Qi Kang, ${ }^{*^{\mathrm{a}}}$ Jiping Ma ${ }^{\mathrm{d}}$ and \\ Lingxin Chen (D) *b,e,c
}

\begin{abstract}
Benzo[a]pyrene (Bap) is one of the main organic pollutants in the atmospheric haze that is rich in fine water drops and particulate matters. The understanding of the Bap's form in water is of great importance to unveil its real biological effects toward the respiratory system. To date, various reports have documented its toxicological effects in the molecular form. Herein, we found that Bap existed as self-aggregated nanoclusters of tunable sizes rather than as dissolved molecules in water and different sized nanoclusters illustrated varied cytotoxicity. These findings indicated that the size, which has been ignored in previous studies, is also a dominant parameter similar to the molecular concentration for determining Bap's cytotoxicity. Polystyrene (PS) nanoparticles, as a model for nanoplastics, could adsorb Bap nanoclusters and serve as carriers that enter the cells. The combination effect interestingly altered the cytotoxicity distinction of Bap of different sizes. The intracellular fate of the nanoparticles and subcellular organelle damages were studied to unveil the mechanisms of cytotoxic distinction. Small Bap nanoclusters entered cells faster than their large counterparts. The Bap of the PS@Bap complex was stably adsorbed on PS at the early stages of endocytosis until it was detached during the lysosomal transport and maturation process. The dissociated Bap may bypass the lysosome pathway and be released into the cytosol with a nanocluster structure or relocate into the endoplasmic reticulum. On the other hand, the detached PS preferred to bind to the mitochondria or be excreted out of the cell via the lysosomal pathway. Moreover, the PS@Bap complex resulted in a significant loss of the mitochondrial membrane potential and induced apoptosis through the mitochondria-involved apoptosis pathway. This study provides a new perspective towards the toxicological mechanism of insoluble hydrophobic organic compounds and reveals the environmental significance of nanoplastics for regulating the biological effects of conventional pollutants.
\end{abstract}

\footnotetext{
${ }^{a}$ College of Chemistry, Chemical Engineering and Materials Science, Key Laboratory of Molecular and Nano Probes, Ministry of Education, Shandong Normal University, Jinan 250014, China. E-mail: kangqi@sdnu.edu.cn

${ }^{b}$ CAS Key Laboratory of Coastal Environmental Processes and Ecological Remediation, Shandong Key Laboratory of Coastal Environmental Processes, Yantai Institute of Coastal Zone Research, Chinese Academy of Sciences, Yantai 264003, China.E-mail: lxchen@yic.ac.cn

${ }^{c}$ Center for Ocean Mega-Science, Chinese Academy of Sciences, Qingdao 266071, China

${ }^{d}$ School of Environmental \& Municipal Engineering, Qingdao University of Technology, Qingdao 266033, China

${ }^{e}$ Laboratory for Marine Biology and Biotechnology, Pilot National Laboratory for Marine Science and Technology, Qingdao 266237, China

$\dagger$ Electronic supplementary information (ESI) available. See DOI: 10.1039/ d0nr06747d

$\$$ These authors contributed equally.
}

\section{Introduction}

Benzo[ $a]$ pyrene (Bap) is a type of hydrophobic organic chemical (HOC) pollutant primarily derived from cigarette smoke, diesel exhaust particulate matters, and byproducts of industrial processes. After vaporizing into the air, Bap interacts with fine water drops and becomes the major organic component of haze. Bap has been proved to rapidly distribute in the organism after being inhaled ${ }^{1}$ and induces oxidative stress and inflammation in pulmonary, ${ }^{2}$ liver, ${ }^{3}$ and neuronal systems. ${ }^{4}$ However, the form in which it interacts with the biological system has seldom been seriously interpreted. In previous laboratory-based toxicity studies, Bap was usually treated as welldispersed, single molecules in aqueous solutions as normal soluble molecules or ions. Recently, several works have revealed that HOCs, including polycyclic aromatic hydrocarbons, heterocyclic compounds, and organometallic compounds, tend to self-aggregate into nanoclusters in aqueous 
media, driven by hydrophobic-lipophilic interactions. ${ }^{5-7}$ This self-aggregation phenomenon universally occurs in water, ${ }^{8}$ atmosphere, ${ }^{9}$ and even specific plant cellular structures. ${ }^{10}$ Because of the strong hydrophobicity of HOCs, the partition coefficients of HOCs between themselves and water can be as high as the order of $10^{9}$. This means that even in a clear, transparent solution at very low HOC concentration (lower than $1 \mu \mathrm{M}$, taking perylene as an example ${ }^{8}$ ), HOCs still exist in the form of nanoclusters.

This discovery hints at the necessity to revisit the toxicity of Bap from the view of nanoparticles. To date, though the uptake, retention, and toxicity of Bap in vivo and in vitro have been widely studied, the real biological effects are incompletely elucidated as conflicting results are usually reported. For example, Kim showed that $1 \mu \mathrm{M}$ Bap had no cytotoxic effect on endometrial carcinoma cells, while Chanyshev demonstrated that Bap evoked a significant level of endometrial carcinoma cells death $(40 \%)$ after 24 h-treatment at the same concentration. $^{11,12}$ These experiments were conducted above the aqueous solubility limit of Bap. ${ }^{13}$ We speculated that Bap existed in the form of nanoclusters and the misunderstanding of Bap's form was probably responsible for the inconsistent results of the previous studies. The biological effects of Bap should be evaluated from the view of nanoparticles rather than a single molecule because the former would offer more comprehensive and realistic information. For soluble molecule samples, only chemical structures and concentrations need to be considered, and these parameters can be exactly described. By contrast, more complicated physicochemical parameters, such as size, shape, surface charge, and surface corona, must be characterized for nanomaterials because they all might determine the nanomaterial's cellular fate concerning internalization mechanism, cellular distribution, metabolism, and excretion. ${ }^{14-20}$ A recent study proved that perylene (a structural analog of Bap) entered cells in the form of nanoclusters. ${ }^{8}$ However, further questions on how different sizes of Bap nanoclusters were produced and how they affected the cellular toxicology need to be answered urgently.

Nanoplastics have recently become a worldwide concern as newly emerging pollutants. They can be produced from macro and microplastics by mechanical breakdown, ${ }^{21}$ light degradation, ${ }^{22}$ and biological digestion processes, ${ }^{23}$ and are then released into water bodies, ${ }^{24,25}$ soil $^{26}$ and atmosphere. ${ }^{27}$ Nanoplastics induce more severe biological risks than microplastics, ${ }^{28,29}$ leading to oxidative stress, DNA damage, and cell malignancy. ${ }^{30,31}$ It is well known that nanoplastics widely exist in waterbodies and soil, and their resultant potential risks toward various organisms and even human health via exposure through the diet route. ${ }^{32-34}$ Currently, there are increasing reports of the presence of nanoplastics in the atmosphere. Nanoplastics generated from fibers of clothes, debris of tyre wear, agricultural PE sheets, contaminated sewage sludge employed as fertilizer, ${ }^{35}$ as well as industrial activities such as the thermal cutting of polystyrene foam, ${ }^{36}$ and $3 \mathrm{D}$ printing $^{37}$ have also been discovered in the atmosphere. However, the toxic effects of nanoplastics on the lungs have rarely been studied to date. Moreover, nanoplastics also induce combined pollution by adsorbing organic compounds (including Bap $^{38,39}$ ) on hydrophobic surface domains. ${ }^{40,41}$ The combination is likely to change the environmental transformation and biological toxicity of Bap. Simultaneously, the adsorption of Bap can also influence the nanoplastics' physiochemical features and cellular responses. The reciprocal effect study is helpful in deeply unveiling the environmental effect of nanoplastics, which has also rarely been studied to date.

Herein, we aim to investigate the size and nanoplasticsadsorbing dependent cytotoxicity of Bap from the view of nanoclusters. Considering that both materials have been widely discovered in the atmosphere and have proved to be harmful to the human lung tissue, ${ }^{42,43}$ lung epithelial cells (A549) were selected as the cell model to mimic a respiratory exposure scenario. It was found that the sizes of Bap nanoclusters with the same concentration could be different, which is attributed to different solution dilution methods. The size of the Bap nanoclusters dramatically affected the cytotoxicity. Small Bap nanoclusters were much more toxic than large ones with the same molecular concentration. Nanoplastics could serve as carriers for Bap nanoclusters and alter the toxicity difference to a moderate level. By utilizing fluorescence labeling and live-cell imaging techniques, the toxicity difference was explained from the perspective of endocytic routes, accumulation levels, and transport dynamics. Moreover, the loss of the mitochondrial membrane potential indicated that the cell toxicity was induced through a mitochondria-involved apoptosis pathway. These findings emphasize the key role of the PAH's form in defining the toxicological effect and mechanism, and the environmental significance of the nanoplastics as an emerging particulate pollutant in the atmosphere.

\section{Results and discussion}

\section{Characterization of Bap and PS@Bap}

In the process of preparing the Bap solutions, we found that the turbidity of the samples increased with the increase in the Bap concentrations, indicating that Bap existed in the form of nanoclusters, considering that it is the dilution process that influences the nanocluster sizes of the organic molecules dissolved in the aqueous medium. Inspired by these findings, Bap nanoclusters were prepared by different solution dilution methods. Briefly, the "one-step" way clusters were prepared by diluting different concentrations of Bap organic stock solutions (DMSO as the solvent; $2.5 \mathrm{mM}, 12.5 \mathrm{mM}, 25 \mathrm{mM}$ ) to the same volume water. The "two-step" method clusters were prepared by the first-round dilution of the Bap stock solution (in DMSO) with water to obtain an aqueous stock solution $(200 \mu \mathrm{M})$, followed by further dilution with water to the same concentration as that in the "one-step" method. The preparation procedure is described in Fig. $\mathrm{S} 1 . \dagger$

The solutions obtained by the "one-step" dilution method were less turbid than those obtained by the "two-step" method at the same concentration (Fig. 1A), indicating that the size of 

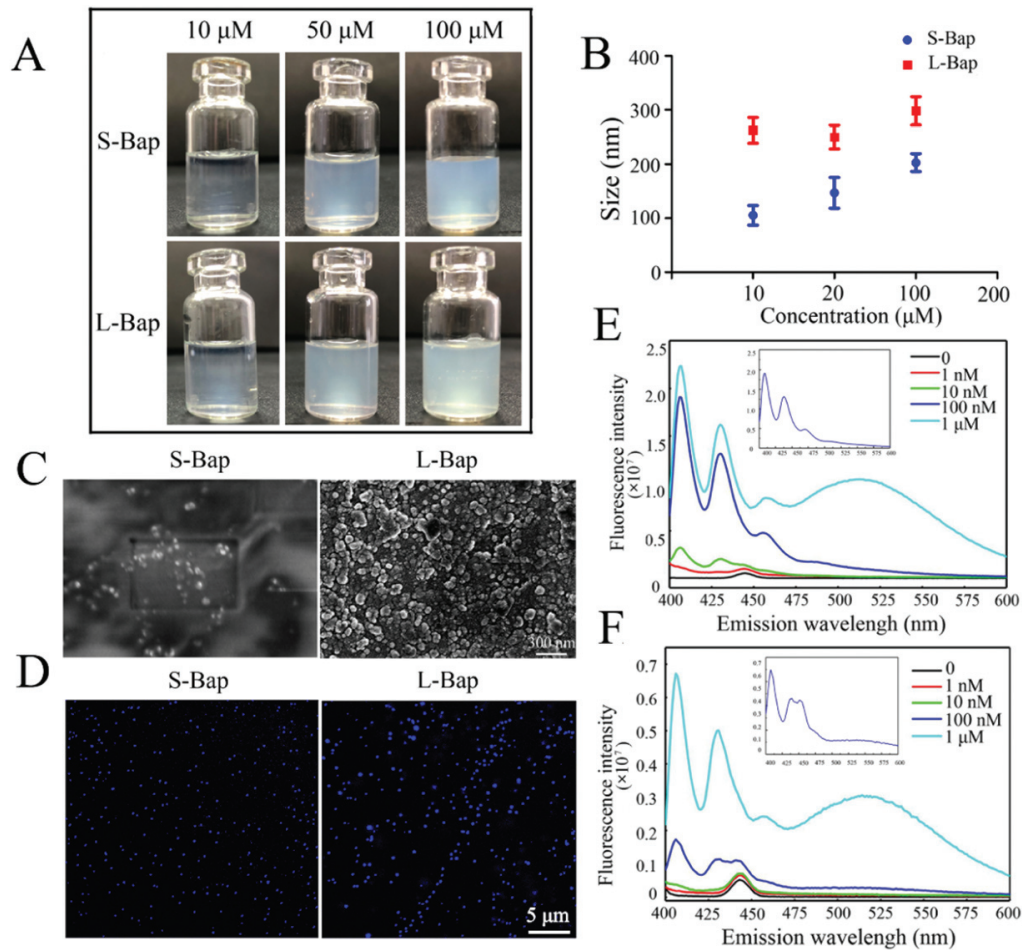

Fig. 1 Photographs (A) and DLS data (B) of S-Bap and L-Bap solutions with different concentrations. SEM images (C) and LSCM images (D, $\lambda_{\text {ex }}$ $405 \mathrm{~nm}, \lambda_{\mathrm{em}} 410-470 \mathrm{~nm}$ ) of the S-Bap samples and L-Bap samples $(1 \mu \mathrm{M})$. Fluorescence spectra of the S-Bap (E) and L-Bap (F) samples at different concentrations excited at $384 \mathrm{~nm}$.

the Bap clusters was different. Four techniques were applied to identify the Bap nanoclusters. First, DLS data revealed that the size of the sample prepared from the "one-step" dilution way was smaller than that obtained from the "two-step" way at each higher test concentration in the range of $10 \mu \mathrm{M}-100 \mu \mathrm{M}$ (Fig. 1B). Accordingly, the samples were named "S-Bap" and "L-Bap", respectively, in the following. Both Bap nanoclusters had a similar weak negative charge, which might account for the phenomenon that the uniform state of the nanoclusters appeared to be relatively stable in the aqueous solution. Considering that the DLS sizes were not detectable at lower concentrations, SEM images were captured as a second way to illustrate the size distinction. As shown in Fig. 1C and Fig. S2, $\uparrow$ the sizes of the two samples $(1 \mu \mathrm{M})$ were about $55 \mathrm{~nm}$ and $142 \mathrm{~nm}$, respectively. Thirdly, by LSCM imaging, Bap was observed to be deposited on the plastic region of the cell culture dish in the form of particulate matter through hydrophobic interactions. A number of blue fluorescent dots rather than a homogenous fluorescent background could be observed on the plastic surface of the cell culture dish. The size difference was apparent (Fig. 1D). Moreover, the fluorescence spectra also verified the form of the nanoclusters. As indicated in Fig. $1 \mathrm{E}$ and $\mathrm{F}$, the emission spectra profiles for both the samples were concentration-dependent. However, the profile evolution features were different. For the S-Bap sample, singlemolecular Bap emission peaks $(400 \mathrm{~nm}, 430 \mathrm{~nm})$ appeared at a low concentration of $10 \mathrm{nM}$. With Bap concentration increasing to as high as $1 \mu \mathrm{M}$, a new peak of the Bap nanoclusters at
475-600 nm appeared. By contrast, for the L-Bap sample, the appearance of single-molecular Bap emission peaks was postponed at $100 \mathrm{nM}$, together with the peak of the Bap clusters. Besides, the fluorescence intensity was nearly 10 times lower than that from the S-Bap sample at the same concentration because of the lower fluorescence quantum yield of the clusters.

To study the combined effect of the Bap nanoclusters and nanoplastics, polystyrene (PS) nanospheres were prepared as nanoplastic models. SEM images (Fig. 2A) showed that the diameter was about $190 \mathrm{~nm}$ and the surface was smooth and clean. After incubation with $1 \mu \mathrm{M}$ Bap solution containing either L-Bap or S-Bap, the PS surfaces became blurry, indicating the adsorption of the Bap nanoclusters. The LSCM imaging results also proved the combination of the two materials. Red fluorescence originated from Nile red-labeled PS nanoparticles and blue fluorescence originated from surface-adsorbed Bap nanoclusters perfectly co-located, as shown in Fig. 2B. The adsorption of Bap nanoclusters did not change the hydrodynamic diameter of bare PS (Fig. S3†); however, it dramatically increased the zeta potential from $-51.4 \mathrm{mV}$ for bare PS to $-28.8 \mathrm{mV}$ (for PS@S-Bap) and $-20.4 \mathrm{mV}$ (for PS@L-Bap), respectively (Fig. 2C). Given that the charge of both Bap nanoclusters and PS was negative, the driving forces for their combination could be hydrophobic interactions rather than electrostatic force (Fig. 2D). The loading capacity of PS toward Bap was further quantitatively examined. Table S1† revealed that with the given amount of PS 


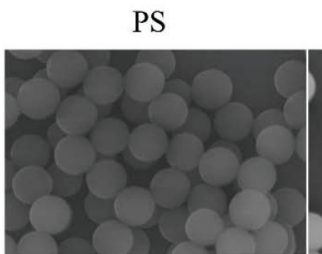

PS@S-Bap
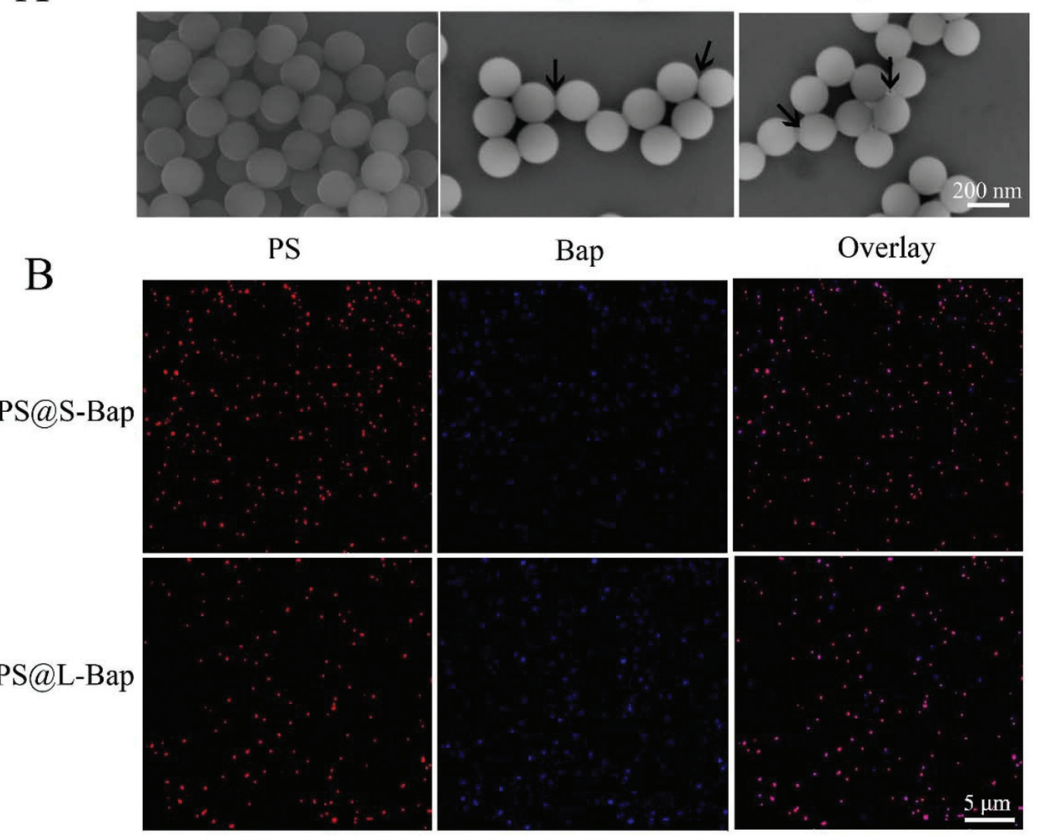

Overlay

$\mathrm{C}$

PS S-Bap L-BapPS@S-BapPS@L-Bap D

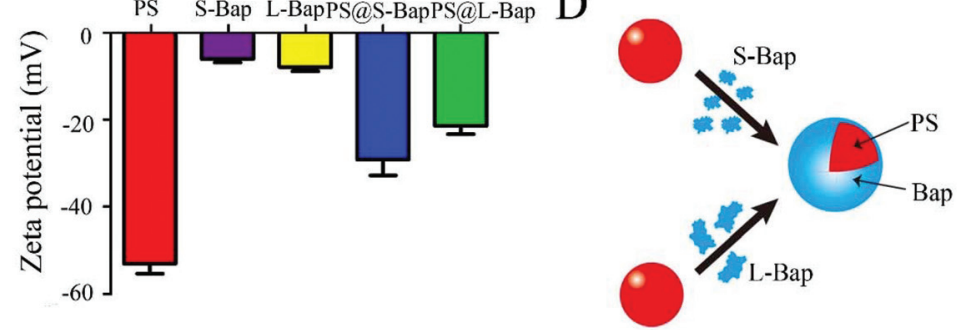

Fig. 2 SEM images of PS, PS@S-Bap, and PS@L-Bap samples; the arrows illustrate the Bap adsorbed on the PS junctions (A). LSCM images of PS@S-Bap and PS@L-Bap samples (B, Nile, $\lambda_{\mathrm{ex}} 594 \mathrm{~nm}, \lambda_{\mathrm{em}} 600-650 \mathrm{~nm}$; Bap, $\lambda$ ex $405 \mathrm{~nm}, \lambda_{\mathrm{em}} 410-470 \mathrm{~nm}$ ). Zeta potential of PS, S-Bap, L-Bap, PS@S-Bap, and PS@L-Bap samples at $1 \mu \mathrm{M}$ Bap concentrations (C). Schematic representation of the formation of the PS@S-Bap and PS@L-Bap samples (D).

nanoparticles $\left(0.5 \mathrm{mg} \mathrm{mL}^{-1}\right)$, the adsorption rates of Bap were more than $90 \%$ for all the tested Bap concentrations $(0.1,1,5$ and $10 \mu \mathrm{M})$. The strong adsorption capacity illustrated great toxicity potential of the complex particles, considering that $1 \mu \mathrm{M}$ Bap was enough to induce significant cytotoxicity.

\section{Cytotoxicity investigation}

The cellular toxicities of Bap nanoclusters, PS, and the complex were examined via the MTT assay. As shown in Fig. 3A, the Bap nanoclusters induced cell death in A549 cells in a size-dependent manner. The S-Bap nanoclusters were much more toxic than those of L-Bap after incubation with the same dosage for $24 \mathrm{~h}$. Bare PS illustrated no detectable negative effect at the tested concentrations (Fig. S5†). Bap nanoclusters bound to PS generated significant cytotoxicity and the cell viability value of the PS@Bap complex was found to be between those induced from free S-Bap and L-Bap nanoclusters. These interesting results implied that co-exposure with PS attenuated the toxicity discrepancy induced by the Bap nanocluster size effect.
The toxicity distinction of the NPs was further elucidated from the perspective of the NPs' intracellular fate via fluorescence imaging. The fluorescence images revealed the appearance of S-Bap in A549 cells in a short period within $10 \mathrm{~min}$. By contrast, the fluorescent signals of L-Bap were scattered in the cytoplasm after incubation for $30 \mathrm{~min}$, showing that the internalization of L-Bap was much slower. The internalization rates of both PS@S-Bap and PS@L-Bap were at an intermediate level between those acquired from L-Bap and S-Bap, indicating that PS could alter the accumulation rate of L-Bap and S-Bap (Fig. 3B). Continuous internalization and accumulation of Bap could be observed with prolonged exposure time. All the samples showed similar increasing trends of fluorescence intensity, which increased rapidly at the earlier stage of cellular uptake (10 min-6 h) and then gradually slowed down afterwards (6-12 h, Fig. 3C and Fig. S6†).

The fluorescence brightness of the LCSM images could not accurately reflect the Bap amount due to the discriminative fluorescence quantum efficiency of differently-sized Bap nanoclusters. Therefore, the toxicity difference was further analyzed 

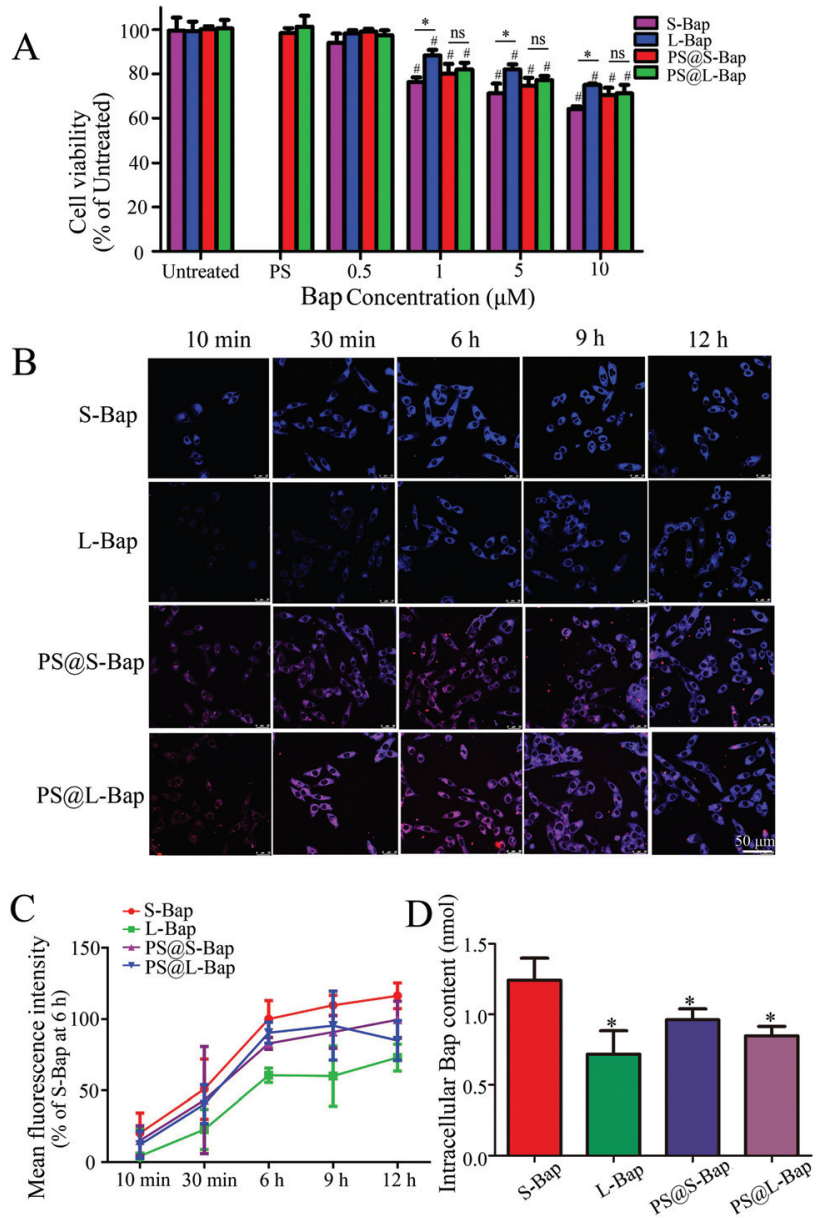

Fig. 3 A549 cells' cytotoxicity assessment after exposure to the indicated concentrations of S-Bap and L-Bap, PS $\left(50 \mu \mathrm{g} \mathrm{mL}^{-1}\right)$, PS@S-Bap (containing $50 \mu \mathrm{g} \mathrm{mL}^{-1} \mathrm{PS}$ ), and PS@L-Bap (containing $50 \mu \mathrm{g} \mathrm{mL}^{-1} \mathrm{PS}$ ). $n=3,{ }^{*} P<0.05$ versus S-Bap. ${ }^{\#} P<0.05$ versus untreated. ns, no significant versus PS@S-Bap (A). Dynamics of accumulation of S-Bap, L-Bap, PS@S-Bap, and PS@L-Bap in A549 cells. Confocal images of A549 cells incubated with $1 \mu \mathrm{M}$ S-Bap, L-Bap, PS@S-Bap (containing $50 \mu \mathrm{g} \mathrm{m}^{-1}$ PS), and PS@L-Bap (containing $50 \mu \mathrm{g} \mathrm{mL}^{-1} \mathrm{PS}$ ) for the indicated time periods (10 $\mathrm{min}, 0.5,6,9$, and $12 \mathrm{~h})\left(\mathrm{B}\right.$, Nile, $\lambda_{\mathrm{ex}} 594 \mathrm{~nm}$, $\lambda_{\mathrm{em}}$ 600-650 nm; Bap, $\lambda_{\mathrm{ex}} 405 \mathrm{~nm}, \lambda_{\mathrm{em}}$ 410-470 nm). Mean fluorescence intensity of internalized $1 \mu \mathrm{M}$ S-Bap, L-Bap, PS@S-Bap (containing $50 \mu \mathrm{g}$ $\mathrm{mL}^{-1} \mathrm{PS}$ ), and PS@L-Bap (containing $50 \mu \mathrm{g} \mathrm{mL}^{-1} \mathrm{PS}$ ) for the indicated time periods (10 $\mathrm{min}, 0.5,6,9$, and $12 \mathrm{~h}$ ) was obtained from the confocal images by analyzing with the Leica Application Suite $X$ software equipped in the Leica SP8 confocal microscope (C). Intracellular Bap content (per $10^{6}$ cells) after exposure to $1 \mu \mathrm{M} \mathrm{S}$-Bap, L-Bap, PS@S-Bap (containing $50 \mu \mathrm{g} \mathrm{mL}^{-1} \mathrm{PS}$ ), and PS@L-Bap (containing $50 \mu \mathrm{g} \mathrm{mL}^{-1} \mathrm{PS}$ ) for $24 \mathrm{~h}$. The Bap content within the cells was extracted by methylbenzene and quantitatively detected via fluorescence intensity measurement by a fluorescence spectrometer, ${ }^{*} P<0.05$ versus S-Bap (D).

in terms of the Bap uptake amount. After incubation for $24 \mathrm{~h}$, intracellular Bap was extracted by methylbenzene and quantitatively detected via fluorescence intensity measurement by a fluorescence spectrometer (Fig. 3D). The results demonstrated that cells exposed to S-Bap showed much more intracellular Bap content compared to those exposed to L-Bap. However, PS could effectively change the uptake amount of both the Bap nanoclusters and result in a similar accumulation of Bap in the A549 cells, which well-explained the MTT results that the toxicity difference between the two types of Bap nanoclusters was considerably reduced following the adsorption of Bap nanoclusters on nanoplastics.

\section{Distinct endocytic mechanisms for Bap nanoclusters and PS@Bap particles}

Since S-Bap and L-Bap showed completely different uptake rates in A549 cells, we suspected that these two samples employed distinct pathways for endocytosis in the cells. To elucidate the endocytosis mechanisms, the cells were preincubated with selective pharmaceutical inhibitors targeting distinct endocytic pathways. $\mathrm{M} \beta \mathrm{CD}, \mathrm{CPZ}, \mathrm{CyD}$, and EIPA were used as inhibitors of caveolae-mediated endocytosis, clathrinmediated endocytosis, phagocytosis, and micropinocytosis, respectively. ${ }^{44-47}$ Compared with the untreated cells, A549 cells pretreated with CPZ showed significantly decreased intracellular signals for S-Bap, whereas pretreating cells with CPZ and CyD resulted in a strong reduction in internalized L-Bap. Interestingly, a significant reduction in the fluorescence intensities was detected by CPZ and CyD endocytotic inhibitors in A549 cells incubated with PS@S-Bap or PS@L-Bap, suggesting that clathrin- and phagocytosis-dependent endocytosis were involved in the internalization of PS@Bap (Fig. 4A and B). Taken together, it could be concluded that S-Bap was mainly internalized by the A549 cells via clathrin-mediated endocytosis while L-Bap was mainly internalized through clathrin and phagocytosis-mediated endocytosis. The different endocytosis mechanism was probably responsible for the differences in the endocytosis amount and the rate of Bap with different sizes. In addition, PS could effectively change the internalization mechanisms for S-Bap and L-Bap to clathrin- and phagocytosis-dependent endocytosis, thus indicating a similar accumulation in A549 cells (Fig. 4C and S7†). This was similar to the size-dependent uptake mechanisms of organic carbon, such as graphene oxide. Small nanosheets were internalized mainly via clathrin-mediated endocytosis and the increase in the size of graphene oxide enhanced the phagocytotic uptake of the nanosheets. ${ }^{48}$ In addition, the conversion of the nanocluster structure to the nanosphere structure following the adsorption of Bap nanoclusters onto PS might offer larger contact surfaces with cell membranes and then lead to Bap entering the cells through different endocytosis pathways.

\section{Intracellular metabolism and excretion for Bap and PS@Bap particles}

We next explored the metabolism and excretion behaviors of Bap and PS@Bap. Owing to multiple fused aromatic ring systems, Bap and metabolites produced by cytochrome P450s and other enzymes were highly fluorescent. However, they showed different fluorescence peak positions because of the structural differences. It has been demonstrated that the $404 \mathrm{~nm}$ peak was characteristic of the parent Bap, whereas the Bap metabolites showed red-shifted peaks in the range of 425-470 nm. Among them, the 425-450 nm band matched the 

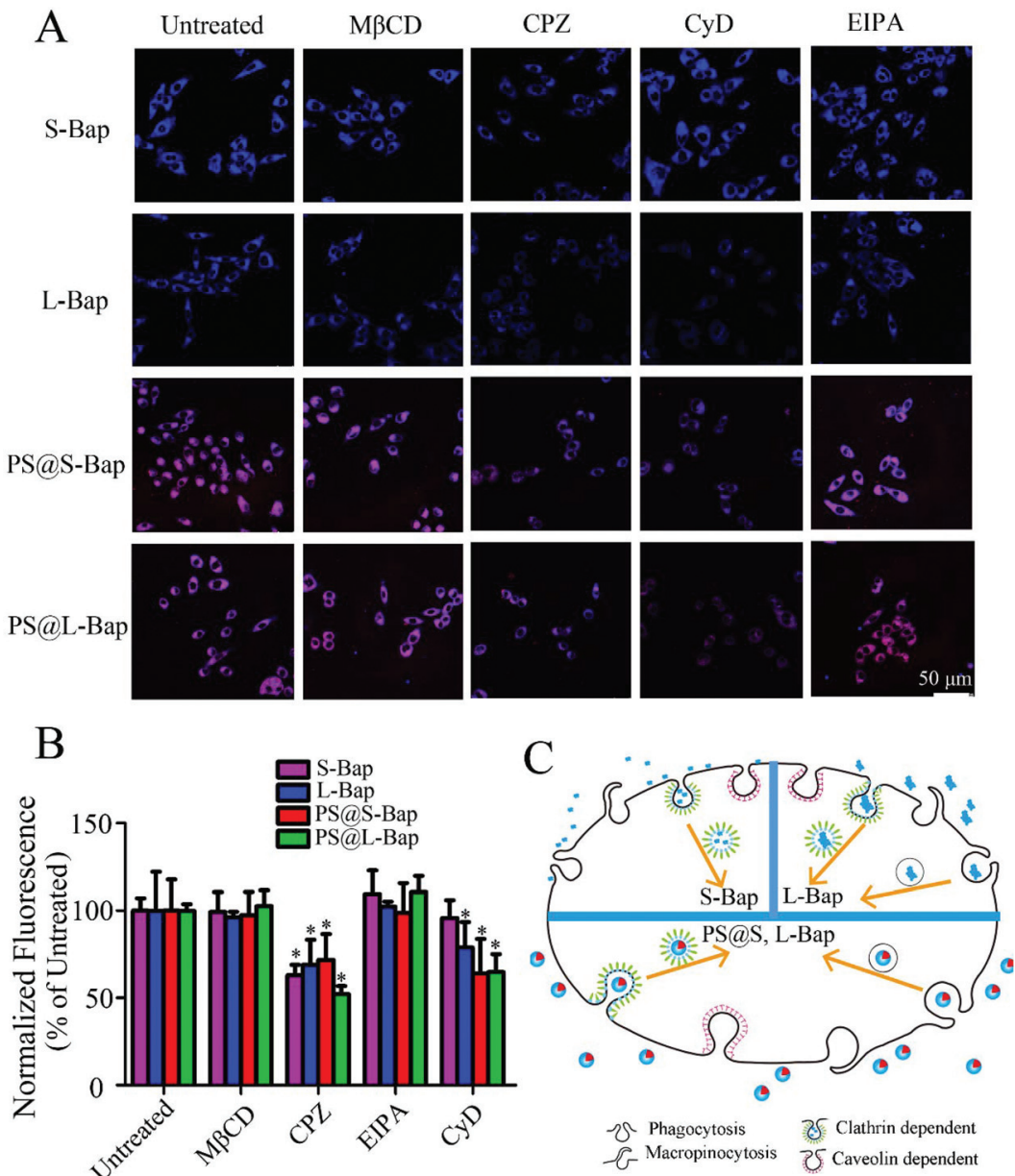

Fig. 4 Effects of different pharmacological inhibitors on the uptake of $1 \mu \mathrm{M} \mathrm{S-Bap,} \mathrm{L-Bap,} \mathrm{PS@S-Bap} \mathrm{(containing} 50 \mu \mathrm{g} \mathrm{mL}-1$ PS), and PS@L-Bap (containing $50 \mu \mathrm{g} \mathrm{mL}^{-1} \mathrm{PS}$ ) by the A549 cells after incubation for $6 \mathrm{~h}$ (A, Nile, $\lambda_{\mathrm{ex}} 594 \mathrm{~nm}, \lambda_{\mathrm{em}} 600-650 \mathrm{~nm}$; Bap, $\lambda_{\mathrm{ex}} 405 \mathrm{~nm}, \lambda_{\mathrm{em}} 410-470 \mathrm{~nm}$ ). Histogram of intracellular fluorescence of Bap, ${ }^{*} P<0.05$ versus untreated (B). Schematic representation of the endocytosis mechanism S-Bap, L-Bap, PS@S-Bap, and PS@L-Bap (C).

major metabolites 3-OH Bap and 9-OH Bap, while the 450-470 nm band corresponded to the secondary metabolite Bap-3,6-dione. This spectral difference has been used for the characterization of in vivo or in vitro metabolic behaviors of Bap by means of scanning the whole wavelength for fluorescence. ${ }^{49-51}$ Based on this, the whole wavelength scanning for the fluorescence of the cell (380-500 nm) was exploited to characterize the Bap metabolism behaviors, relying on the maximum fluorescence peak position difference of Bap (400 nm) and the metabolites (430 nm and $460 \mathrm{~nm}$ ).

As shown in Fig. 5A and B, the fluorescence intensity ratio $\left(I_{460 \mathrm{~nm}} / I_{400 \mathrm{~nm}}\right)$ increased on prolonging the incubation time, indicating the chemical transformation of Bap. S-Bap displayed a much faster metabolite rate compared to the larger counterparts, which might be attributed to S-Bap possessing a larger surface area that increased the probability of interacting with related metabolite enzymes (cytochrome P450 and epoxide hydrolase) and receptors (aryl hydrocarbon receptors). Interestingly, the cells exposed to S, L-Bap showed a decrease in the fluorescence intensity and metabolite rate after $24 \mathrm{~h}$, which most likely corresponded to Bap excretion in the cells. Bap in the PS-bound state underwent relatively slower metabolism progress compared to free Bap probably because PS altered the location or duration of Bap in the metabolism environment (Fig. 5A and B). As shown in Fig. S8, $\dagger$ at the earlier stage of cellular uptake, Bap was in a "captured" state, as revealed by the fact that PS and the fluorescence signals mostly remained colocalized. After incubation for $6 \mathrm{~h}$, separate fluorescence of Bap (blue) could be apparently monitored, indicating that the appearance of free Bap in the cytosol lagged dramatically. Besides, the decline in the Bap fluorescence in the cells incubated with PS@Bap was faster compared to those incubated with the Bap nanocluster samples, suggesting PS as a Bap carrier might accelerate Bap excretion from cells in the combination NP form (Fig. S9†). It is noteworthy that the fluorescence intensity ratio $\left(I_{460 \mathrm{~nm}} / I_{400 \mathrm{~nm}}\right)$ of Bap in the cells exposed to PS@Bap still increased at $24 \mathrm{~h}$, which was speculated to be due to the mutual combined results of metabolism and excretion. Taken together, PS could significantly alter the metabolism and excretion behaviors of 

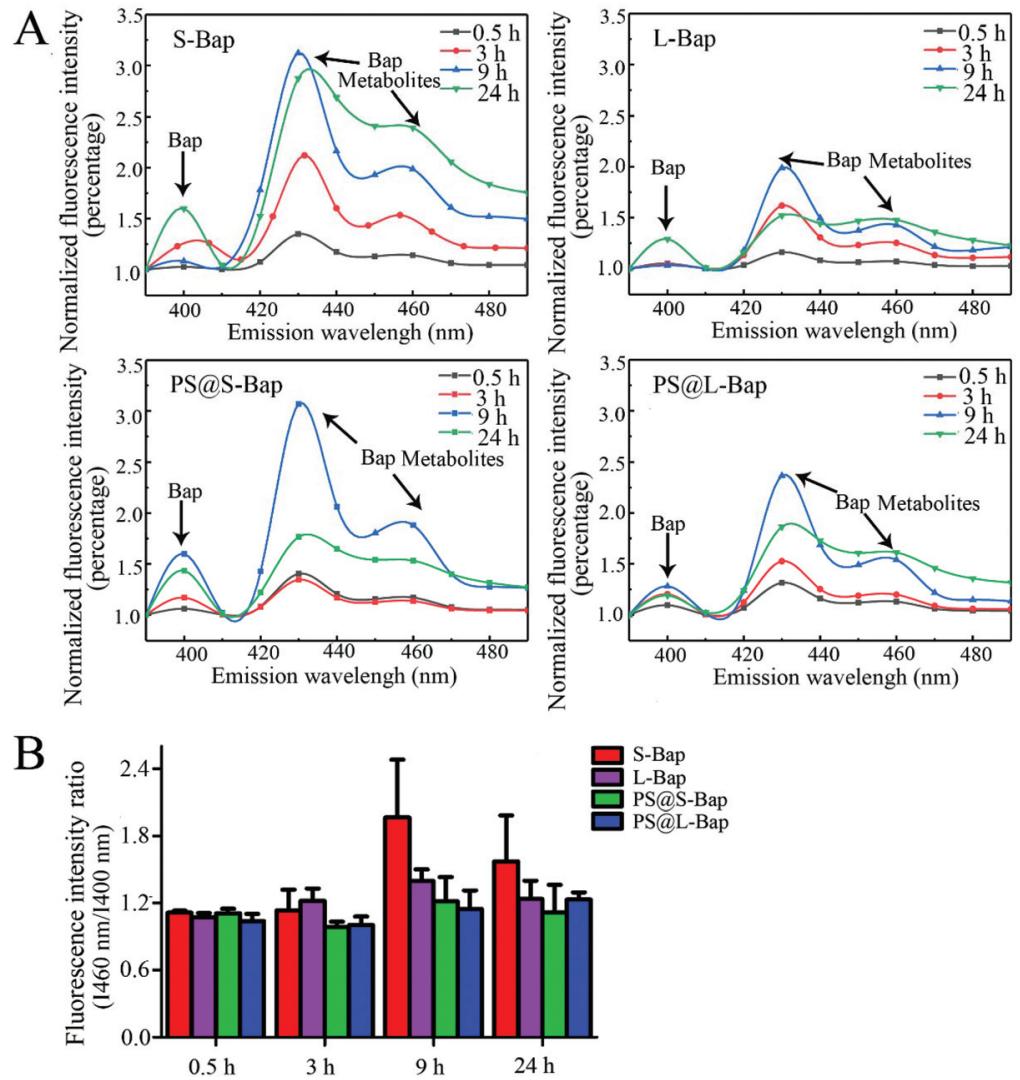

Fig. 5 Graphical representation of the whole wavelength scan performed on $1 \mu \mathrm{M} \mathrm{S-Bap,} \mathrm{L-Bap,} \mathrm{PS@S-Bap} \mathrm{(containing} 50 \mu \mathrm{mL}{ }^{-1} \mathrm{PS}$ ), and PS CL-Bap (containing $50 \mu \mathrm{g} \mathrm{mL}^{-1}$ PS) exposed A549 cells for the indicated time periods $(0.5,3,9$, and $24 \mathrm{~h}$ ). The $400 \mathrm{~nm}$ peak is characteristic of Bap, whereas the Bap metabolites show peaks in the range of $430 \mathrm{~nm}-470 \mathrm{~nm}$ (A, $\lambda_{\mathrm{ex}} 384 \mathrm{~nm}, \lambda_{\mathrm{em}} 390-490 \mathrm{~nm}$ ). Histogram of the fluorescence intensity ratio $\left(I_{470 \mathrm{~nm}} / I_{400} \mathrm{~nm}\right)$ of A549 cells incubated with S-Bap, L-Bap, PS@S-Bap, and PS@L-Bap for the indicated time periods (B).

S-Bap and L-Bap, and further reduce the toxicity difference between the two types of Bap nanoclusters.

\section{Intracellular delivery and localization of Bap nanoclusters or PS@Bap in A549 cells}

To dynamically track the intracellular delivery of the Bap nanoclusters or PS@Bap, we investigated whether endocytic Bap nanoclusters or PS@Bap adopted the endosome/lysosome pathway. Endosomes and lysosomes were labeled with LysoTracker, ${ }^{52}$ then the colocalization among Bap, PS@Bap, and endosome/lysosome was imaged at 1, 3, 6 and $12 \mathrm{~h}$ (Fig. 6A). The ratio of Bap that was localized into the endosome/lysosome in the cells exposed to S, L-Bap reached a peak value at $3 \mathrm{~h}$ and gradually declined afterward (Fig. S11 $\dagger$ ). For PS@Bap, the strong colocalization of LysoTracker, PS, and Bap fluorescence signals indicated that the endocytic PS@Bap particles fused with the endosome/lysosome and Bap were retained on the nanoparticle surface at the early stages of endocytosis uptake (1-3 h). Upon $6 \mathrm{~h}$ of internalization, PS maintained high colocalization with the LysoTracker and only the blue fluorescence associated with Bap was isolated, indicating that Bap was desorbed from PS during lysosomal maturation. A decline in the PS fluorescence was found in the cytoplasm probably because it was excreted out of the cell, whereas
Bap was still detectable inside the cells at $12 \mathrm{~h}$. These observations suggested that Bap was stably adsorbed on PS throughout the internalization process and was subsequently desorbed from PS along with lysosomal transport and maturation. The dissociated Bap might bypass the lysosome pathway and be released into the cytosol, whereas the detached PS was excreted out of the cell via the lysosomal pathway.

For a detailed investigation of the subcellular localization of Bap or PS@Bap after internalization into the cells, the cells were stained with the corresponding organelle-specific dyes. As observed by the LSCM images, most Bap existed in the endoplasmic reticulum when the cells were incubated with $\mathrm{S}$, L-Bap for 6 h. For A549 cells incubated with PS@Bap, Bap desorbed from PS was more inclined to enter into the endoplasmic reticulum, while PS accumulated only negligibly in the endoplasmic reticulum (Fig. 6B). The high concentration of Bap in endoplasmic reticulum had been confirmed by $\mathrm{Ali},{ }^{46}$ which might due to the greater ability of the endoplasmic reticulum to adsorb and dissolve lipophilic Bap, considering that it is the main site for the synthesis and transportation of lipids.

As shown in Fig. 6C, a large proportion of PS appeared in the mitochondria when the cells were incubated with PS for $6 \mathrm{~h}$ (Fig. S12 $\dagger$ ). By contrast, there was no significant accumulation of Bap in the mitochondria after exposure to S, L-Bap. 


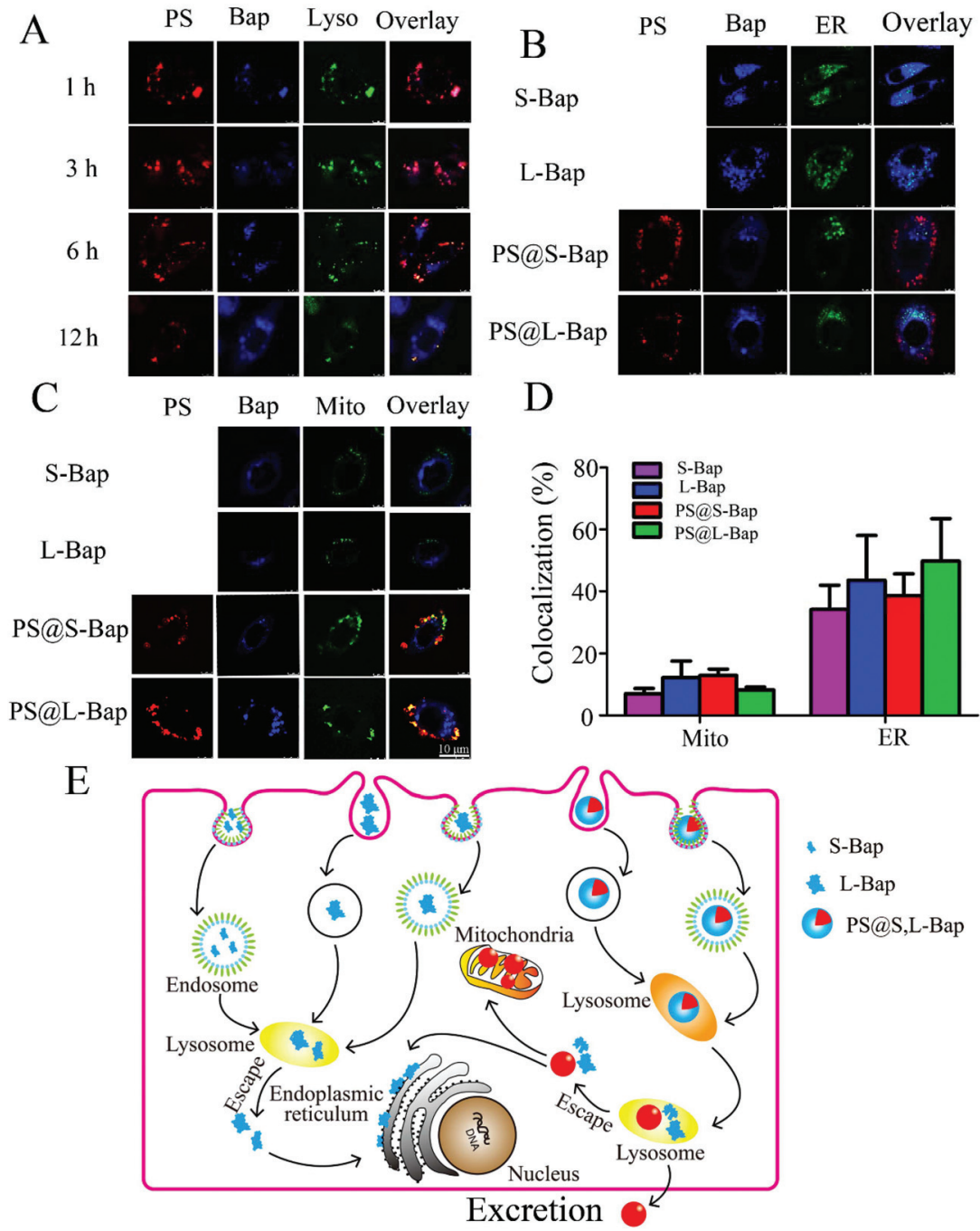

Fig. 6 Colocalization between the lysosome, mitochondria, endoplasmic reticulum and S-Bap, L-Bap, PS@S-Bap, and PS@L-Bap. A549 cells expressing the lysosome (Lyso, green, A), endoplasmic reticulum (ER, green, B), abd mitochondria (Mito, green, C) were incubated with $1 \mu$ M S-Bap, L-Bap, PS@S-Bap (containing $50 \mu \mathrm{g} \mathrm{mL}^{-1} \mathrm{PS}$ ), and PS@L-Bap (containing $50 \mu \mathrm{g} \mathrm{mL} \mathrm{L}^{-1} \mathrm{PS}$ ) and imaged by confocal microscopy (Mito-Tracker, Lyso-Tracker, and ER-Tracker, $\lambda_{\text {ex }} 488 \mathrm{~nm}, \lambda_{\text {em }} 500-540 \mathrm{~nm}$; Nile, $\lambda_{\text {ex }} 594 \mathrm{~nm}, \lambda_{\mathrm{em}} 600-650 \mathrm{~nm}$; Bap, $\lambda_{\mathrm{ex}} 405 \mathrm{~nm}, \lambda_{\mathrm{em}} 410-470 \mathrm{~nm}$ ) at the indicated time points. The percentages of Bap colocalized with the mitochondria and endoplasmic reticulum were quantified using the ImageJ software. (D). Schematic representation of intracellular trafficking and their localization of S, L-Bap and PS@S, L-Bap in the A549 cells (E).

For PS@Bap, the detached PS fluorescence showed high colocalization with the mitochondria, whereas very low colocalization signals between Bap and MitoTracker were detected. Similar biodistribution into the mitochondria was observed in some cell types using negatively charged PS NPs and organic carbon. ${ }^{53-55}$ We speculated that there may be the following factors: (1) In the acidic lysosome environment, the NPs surface is protonated and interacts with the negatively charged mitochondrial membrane through electrostatic interactions. (2) During the lysosomal transport process, the surface of the nanoplastics is covered by protein halos that could target and locate into the mitochondria, such as vacuolar protein sorting 13 A (VPS13) ${ }^{56}$ and Rapamycin Complex 1 (mTORC1). ${ }^{57}$ It had been reported that when NPs are transported through various endomembrane organelles during the trafficking process, specific proteins in these organelles are adsorbed on the NPs surface and the specific protein corona formed during intracellular trafficking affects the subsequent intracellular transportation and localization of NPs. ${ }^{58}$ Collectively, Bap nanoclusters or dissociated Bap from PS@Bap are mainly distributed in the endoplasmic reticulum and lysosomes, while the PS presented in the mitochondria or are excreted via the lysosomal pathway (Fig. 6E).

In addition, it can be deduced that Bap can enter the cells by endocytosis in the form of nanoclusters ${ }^{8}$ and can then gradually dissolve in a dynamic process. When the Bap nanocluster is present in a hydrophobic microenvironment such as a sub-organelle phospholipid membrane and a lipid droplet, ${ }^{49}$ it tends to dissolve into the molecular form. By contrast, when it enters specific sub-organelles and is exposed to a hydrophilic microenvironment (assures the function of metabolic enzymes or maintains the acidic metabolic environment), the 
dissolution might be slowed down and Bap might exist as a cluster for a long time.

\section{Cell apoptosis}

Mitochondrial colocalization results showed that there was only a negligible accumulation of Bap within the mitochondrial matrix after Bap nanocluster or PS@Bap treatment. However, our results indicated a considerable impact of Bap on the mitochondrial function, which was also verified by Ali. ${ }^{49}$ We employed the green fluorescent dye JC-1 to monitor the effects of Bap nanoclusters and PS@Bap on the mitochondrial function. In the control cells, the fluorescent images displayed a predominantly red fluorescence of the JC- 1 aggregates after $24 \mathrm{~h}$ incubation, indicating healthy mitochondria. On the other hand, the formation of JC- 1 aggregates was diminished and obvious diffused green fluorescence was visible following the exposure to Bap nanoclusters or PS@Bap for $24 \mathrm{~h}$. We found that cells exposed to S-Bap presented a more pronounced fluorescence shift compared to that of L-Bap.
However, when the cells were exposed to PS@S-Bap and PS@L-Bap, the level of fluorescence shift was significantly higher than that of L-Bap but lower than that of S-Bap (Fig. 7A). To further confirm the above results, A549 cells were treated with Bap nanoclusters or PS@Bap and stained with JC-1, followed by FCM (Fig. 7B). The FCM results showed control cells with well-polarized mitochondria localized in the upper region of the plot, revealing no mitochondrial damage. A549 cells treated with S-Bap, L-Bap, and PS@S, L-Bap presented a significant loss in the red fluorescence and a downward shift, suggesting the loss of MMP and mitochondrial damage. Also, the shifting intensity was consistent with the results obtained from LSCM. The results obtained from microscopy or cytofluorimetric pattern suggested a significant change in the membrane potential of mitochondria following the exposure to Bap nanoclusters or PS@Bap. Small Bap nanoclusters induced more serious mitochondrial damage than large ones, whereas PS could homogenize the mitochondrial toxicity difference to a moderate level.
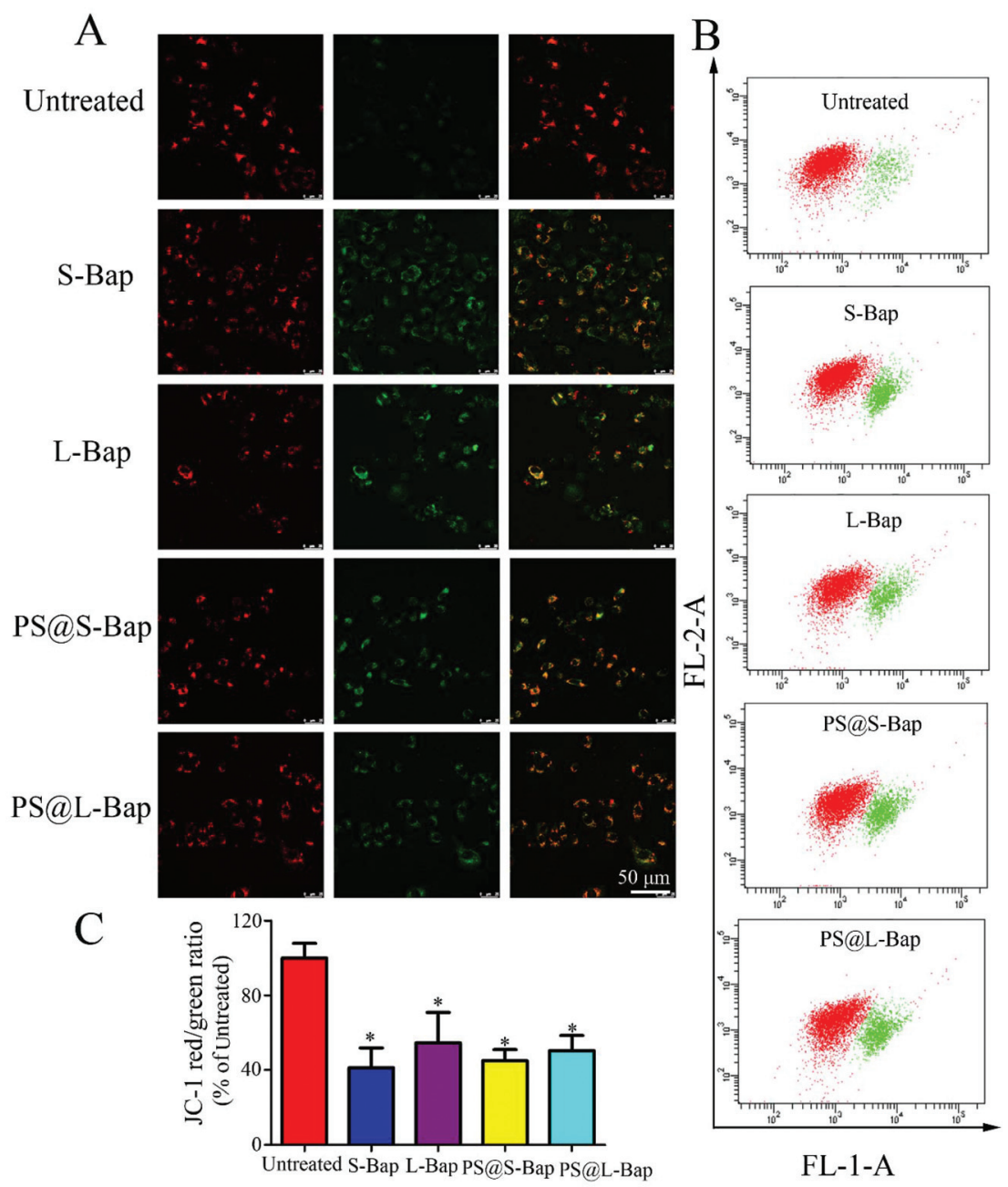

Fig. 7 A549 cells stained with JC-1 after exposure to $1 \mu \mathrm{M} \mathrm{S-Bap,} \mathrm{L-Bap,} \mathrm{PS@S-Bap} \mathrm{(containing} 50 \mu \mathrm{g} \mathrm{mL} \mathrm{m}^{-1} \mathrm{PS}$ ), and PS@L-Bap (containing $50 \mu \mathrm{g}$ $\mathrm{mL}^{-1} \mathrm{PS}$ ) for $24 \mathrm{~h}$ were detected by CLSM (A, JC-1 aggregates, $\lambda_{\mathrm{ex}} 594 \mathrm{~nm}, \lambda_{\mathrm{em}} 600-650 \mathrm{~nm}$; JC-1 monomer, $\left.\lambda_{\mathrm{ex}} 488 \mathrm{~nm}, \lambda_{\mathrm{em}} 500-540 \mathrm{~nm}\right)$ and $\mathrm{FCM},{ }^{*} P<0.05$ versus untreated $(\mathrm{B}$ and $\mathrm{C})$. 


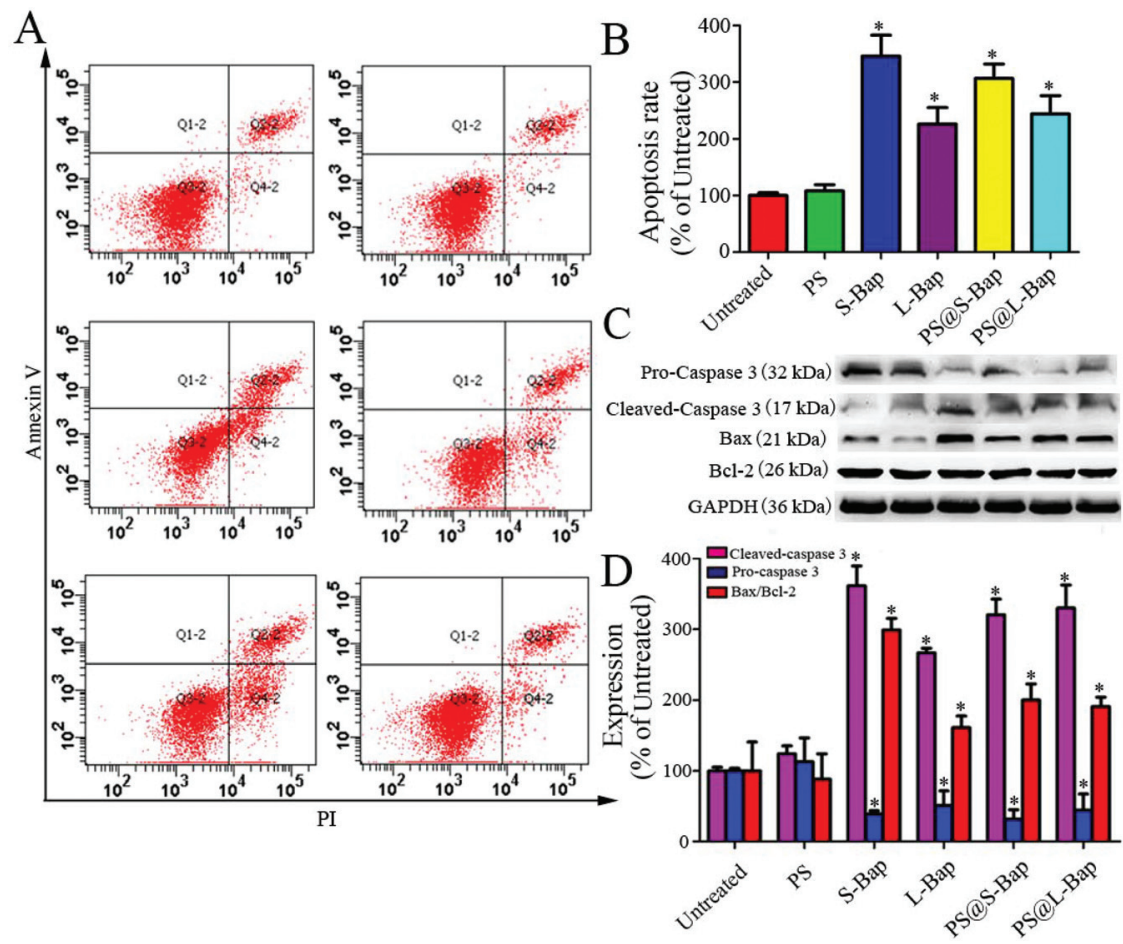

Fig. 8 Cell apoptosis was analyzed by FCM following exposure to $1 \mu \mathrm{M} \mathrm{S-Bap,} \mathrm{L-Bap,} \mathrm{PS@S-Bap} \mathrm{(containing} 50 \mu \mathrm{g} \mathrm{mL} \mathrm{m}^{-1} \mathrm{PS}$ ), and PS@L-Bap (containing $50 \mu \mathrm{g} \mathrm{mL}^{-1} \mathrm{PS}$ ) for $24 \mathrm{~h}$ (A and B). The cells were treated with the indicated concentrations of S-Bap, L-Bap, PS@S-Bap, and PS@L-Bap for $24 \mathrm{~h}$. The total cell lysates were prepared and the protein levels of Caspase 3, Bax, and Cleaved-Caspase 3 were detected by western blot analysis. ${ }^{*} P<0.05$ versus untreated ( $C$ and $\left.D\right)$.

The reduction in the mitochondrial membrane potential is a key biological outcome that further induces impairment of the mitochondrial function, a subsequent decrease in the ATP levels, and the onset of apoptosis. Thus, we explored cell apoptosis by FCM with the annexin V/PI assay following exposure to the Bap nanoclusters or PS-Bap for $24 \mathrm{~h}$ (Fig. 8A). The results showed that the apoptotic rates were $10.43 \%, 33.30 \%, 21.83 \%$, $30.80 \%$, and $23.57 \%$ when A549 cells were treated with PS, S-Bap, L-Bap, PS@S-Bap, and PS@L-Bap for 24 h (Fig. 8B), respectively. There was no significant difference between untreated and PS alone treated cells. By contrast, Bap induced significant apoptosis in a size-dependent manner. The apoptosis rates of PS@S-Bap and PS@L-Bap were in between those of S-Bap and L-Bap, indicating that PS could considerably homogenize the size-dependent toxicity of Bap.

During apoptosis, Bcl-2, one of the main mitochondrialassociated antiapoptotic proteins, effectively prevents cytochrome $\mathrm{c}$ from releasing into the cytoplasm from the mitochondria, ${ }^{59}$ while $\mathrm{Bcl}-2$ associated $\mathrm{X}$ apoptosis regulator (Bax) creates pores in the outer mitochondrial membrane, which results in an increase in the mitochondrial permeability. ${ }^{60}$ Once the mitochondrial damage occurs, pro-Caspase 3 recruits molecules and promotes their cleavage to an active form, which is known as the executor of apoptosis. ${ }^{61}$ Our results showed that treatment with Bap nanoclusters or PS@Bap remarkably up-regulated the expression of $\mathrm{Bcl}-2$ and Caspase 3 , and down-regulated the ratio of $\mathrm{Bax}$ and $\mathrm{Bcl}-2$ and the clea- vage of Caspase 3 (Fig. $8 \mathrm{C}$ and D). All the results indicated that PS-Bap and Bap nanoclusters induced a significant loss in the mitochondrial membrane potential, which might further trigger the apoptosis of A549 cells by activating the mitochondrial proapoptotic factors such as Caspase, Bax, and Bcl-2.

\section{Conclusion}

In the study, we verified that Bap existed as nanoclusters in the aqueous solution with tunable sizes. Bap nanoclusters induced cytotoxicity in A549 cells in a size-dependent manner at the same mass dosage. Nanoplastics could adsorb Bap nanoclusters and alter the toxicity difference to a moderate level by totally altering the intracellular behaviors and toxic mechanism of the Bap nanoclusters, which was proved by fluorescent labeling techniques and live-cell imaging method. This study will open a new avenue for future investigations on the environmental fate and toxicological mechanism of PAHs, and provide basic information and evidence for combined toxicological effects.

\section{Materials and methods}

\section{Chemicals and reagents}

Bap, Nile red, pharmaceutical inhibitors Methyl- $\beta$-cyclodextrin (M $\beta \mathrm{CD})$, Chlorpromazine (CPZ), 5 -( $N$-ethyl- $N$-isopropyl)-amilor- 
ide (EIPA), and Cytochalasin D (CyD) were purchased from Sigma. A549 cells were purchased from Cell Bank, Shanghai Institutes for Biological Sciences. Roswell Park Memorial Institute (RPMI) 1640 Medium, fetal bovine serum (FBS), phosphate buffer solution (PBS), and 5\% trypsin were purchased from Gibco. Streptomycin, penicillin, 5,5',6,6'-tetrachloro1,1',3,3'-tetraethylbenzimidazolylcarbocyanine iodide (JC-1) assay kit, Radio-Immunoprecipitation assay (RIPA) lysis buffer, BCA protein assay kit, sodium dodecyl sulfate polyacrylamide gel electrophoresis (SDS-PAGE), 3-(4,5-dimethyl-2-thiazolyl)2,5-diphenyl-2- $H$-tetrazolium bromide (MTT), goat anti-mouse HRP conjugated IgG, and goat anti-rabbit HRP conjugated IgG were purchased from Beyotime Biotechnology. Dimethyl sulfoxide (DMSO) and methylbenzene was purchased from Sinopharm Chemical Reagent Company. MitoTracker ${ }^{\mathrm{TM}}$ Green, ER TrackerTM Green, and LysoTracker ${ }^{\mathrm{TM}}$ Green were purchased from Thermo Scientific. Annexin V-FITC/PI Apoptosis Detection kit was purchased from BD. Caspase 3, Bax, Bcl-2, Cleaved Caspase 3, and anti-GAPDH Ab were purchased from Cell Signaling Technology. Ultrapure water was used throughout this work.

\section{Preparation of Bap nanoclusters via different solution dilution approaches}

Bap nanoclusters with different sizes were prepared via different solution dilution approaches. A stock solution of $50 \mathrm{mM}$ Bap was prepared by using DMSO as the solvent and stored at $4{ }^{\circ} \mathrm{C}$. Small Bap nanoclusters (S-Bap) were prepared by diluting $4 \mu \mathrm{L}$ of different concentrations (0.025, 0.25, 2.5, 5, $12.5,25$, and $50 \mathrm{mM}$ ) of Bap solutions (DMSO as solvent) with $996 \mu \mathrm{L}$ water. Large Bap nanoclusters (L-Bap) were prepared by diluting $4 \mu \mathrm{L}$ of the stock solution (50 mM) 250 times using ultrapure water, which was then diluted with water to $100 \mathrm{nM}$, $1 \mu \mathrm{M}, 10 \mu \mathrm{M}, 20 \mu \mathrm{M}, 50 \mu \mathrm{M}, 100 \mu \mathrm{M}$, and $200 \mu \mathrm{M}$ concentrations.

\section{Synthesis of PS nanoparticles}

Styrene (St) was first purified to remove the inhibitors. $20 \mathrm{~mL}$ St and $15 \mathrm{~mL} \mathrm{NaOH}(1 \mathrm{M})$ were added into the $100 \mathrm{~mL}$ separation funnel. After shaking the separation funnel, the upper layer was collected and washed with $\mathrm{NaOH}$ three times, then similarly washed with $30 \mathrm{~mL}$ ultrapure water twice. The upper liquid was poured into an Erlenmeyer flask, $\mathrm{NaSO}_{4}$ was added until the solid particles were not agglomerated, and was stored for refrigeration. Then, sodium dodecylsulfate (SDS, $0.2 \mathrm{~g}$ ) and potassium persulfate (KPS, $0.15 \mathrm{~g}$ ) were added to a mixture of $40 \mathrm{~mL}$ ethanol and $100 \mathrm{~mL}$ water in a $250 \mathrm{~mL}$ two-necked flask equipped with a reflux condenser and a Teflon-coated magnetic stirring bar. The reaction mixture was stirred for $30 \mathrm{~min}$ under nitrogen atmosphere. It was then stirred at $70{ }^{\circ} \mathrm{C}$ for $10 \mathrm{~min}$ and $7 \mathrm{~mL}$ of purified St was added to the flask. The reaction was continued with heating at $70^{\circ} \mathrm{C}$ for another $10 \mathrm{~h}$ under a nitrogen atmosphere. PS was washed 3 times via centrifugation in water (16000 rpm for $30 \mathrm{~min}$ ) to remove the residual reactants. Before the experiments, the nanoparticle suspensions were dispersed by sonication.

\section{Fluorescent labeling of PS nanoparticles}

PS nanoparticle solution $\left(0.5 \mathrm{mg} \mathrm{mL} \mathrm{m}^{-1}, 1 \mathrm{~mL}\right)$ was incubated with $2 \mu \mathrm{L}$ Nile red $\left(5 \times 10^{-4} \mathrm{M}\right)$ and stirred for $2 \mathrm{~h}$. The solutions were centrifuged at $16000 \mathrm{rpm}$ for $30 \mathrm{~min}$ and washed with ultrapure water three times to obtain optically labeled PS nanoparticles.

\section{Preparation of PS@Bap composite particles}

Different concentrations of two kinds of Bap nanoclusters were prepared and mixed in a certain proportion of PS nanoparticles (or optically labeled PS nanoparticles). The solutions were centrifuged at $16000 \mathrm{rpm}$ for $30 \mathrm{~min}$ and washed with ultrapure water three times to obtain PS@S-Bap and PS@L-Bap composite particles. After centrifugation at $16000 \mathrm{rpm}$ for 30 min and washing with ultrapure water three times, the supernatants were extracted by adding methylbenzene and stirring for $30 \mathrm{~min}$. The content of Bap in the supernatants was measured by a fluorescence spectrometer to determine the amount of Bap adsorbed on the surface of PS.

\section{Characterization of Bap nanoclusters, PS nanoparticles, and PS@Bap composite particles}

The fluorescence spectra of S-Bap and L-Bap were recorded using a FluoroMax-4 spectrophotometer (HORIBA Scientific, USA). The solutions $(1.0 \mathrm{~mL})$ in a $4 \mathrm{~mL}$ tube were excited at $384 \mathrm{~nm}$ and the emission spectra were recorded in the range of 400-560 nm. The morphology of the S, L-Bap nanoclusters, PS nanoparticles, and PS@Bap composite particles was observed on an S-4800 field emission scanning electron microscope (SEM, Hitachi, Japan) and a Leica SP8 confocal microscope (CLSM, Leica, Germany, Nile, $\lambda_{\text {ex }} 594 \mathrm{~nm}, \lambda_{\mathrm{em}}$ 600-650 nm; Bap, $\lambda_{\text {ex }} 405 \mathrm{~nm}, \lambda_{\text {em }}$ 410-470 nm). The mean hydrodynamic diameter and zeta potential of the Bap nanoclusters, PS nanoparticles, and PS@Bap composite particles were measured using a Zetasizer NanoZS90 (Malvern Instruments, U.K.).

\section{Statistical analysis}

Data were represented as mean \pm standard deviation. Statistical analysis was performed by one-way analysis of variance (ANOVA), followed by Dunnett's test. $P<0.05$ was considered to be statistically significant.

\section{Author contributions}

L. C. and Q. K. designed the experiment. Y. J., Y. W., and D. S. analyzed the data and wrote the first draft. All the authors contributed extensively to the conduction of the experiments and the revision of this paper.

\section{Conflicts of interest}

The authors declare no competing financial interests. 


\section{Acknowledgements}

This study is financially supported by the National Nature Science Foundation of China (no. 42076199, 21976209, 21874083, 21575080, 21976099, 81573393), the program of Youth Innovation Promotion Association, CAS (Grant 2017256), the Instrument Developing Project of the Chinese Academy of Sciences (YZ201662), and Taishan Scholar Project Special Funding (no. ts20190962).

\section{References}

1 A. Ramesh, D. B. Hood, F. Inyang, M. Greenwood, A. M. Nyanda, A. E. Archibong and M. E. Knuckles, Polycyclic Aromat. Compd., 2002, 22, 969-980.

2 S.-Y. Zhang, D. Shao, H. Liu, J. Feng, B. Feng, X. Song, Q. Zhao, M. Chu, C. Jiang, W. Huang and X. Wang, Redox Biol., 2017, 13, 459-469.

3 L. Yuan, J. Liu, H. Deng and C. Gao, J. Agric. Food Chem., 2017, 65, 9763-9773.

4 E. A. Khan, L. B. Bertotto, K. Dale, R. Lille-Langoy, F. Yadetie, O. A. Karlsen, A. Goksoyr, D. Schlenk and A. Arukwe, Environ. Sci. Technol., 2019, 53, 7036-7044.

5 T. Tachikawa, H.-R. Chung, A. Masuhara, H. Kasai, H. Oikawa, H. Nakanishi, M. Fujitsuka and T. Majima, J. Am. Chem. Soc., 2006, 128, 15944-15945.

6 D. Chen, T. S. Totton, J. W. J. Akroyd, S. Mosbach and M. Kraft, Carbon, 2014, 67, 79-91.

7 M. Rapacioli, F. Calvo, F. Spiegelman, C. Joblin and D. J. Wales, J. Phys. Chem. A, 2005, 109, 2487-2497.

8 X. Guo, X. Jin, X. Lv, Y. Pu and F. Bai, Environ. Sci. Technol., 2015, 49, 7926-7933.

9 M. Rapacioli, F. Calvo, C. Joblin, P. Parneix, D. Toublanc and F. Spiegelman, Astron. Astrophys., 2006, 460, 519-531.

10 E. Wild, J. Dent, G. O. Thomas and K. C. Jones, Environ. Sci. Technol., 2006, 40, 907-916.

11 J. Y. Kim, J. Y. Chung, J. E. Park, S. G. Lee, Y. J. Kim, M. S. Cha, M. S. Han, H. J. Lee, Y. H. Yoo and J. M. Kim, Endocrinology, 2007, 148, 5112-5122.

12 M. D. Chanyshev, O. A. Koval, A. A. Nushtaeva and L. F. Gulyaeva, J. Biochem. Mol. Toxicol., 2019, 33, e22314.

13 R. S. Pearlman, S. H. Yalkowsky and S. Banerjee, J. Phys. Chem. Ref. Data, 1984, 13, 555-562.

14 J. Ma, R. Liu, X. Wang, Q. Liu, Y. Chen, R. P. Valle, Y. Y. Zuo, T. Xia and S. Liu, ACS Nano, 2015, 9, 10498-10515.

15 K. F. Chung, J. Seiffert, S. Chen, I. G. Theodorou, A. E. Goode, B. F. Leo, C. M. McGilvery, F. Hussain, C. Wiegman, C. Rossios, J. Zhu, J. Gong, F. Tariq, V. Yufit, A. J. Monteith, T. Hashimoto, J. N. Skepper, M. P. Ryan, J. Zhang, T. D. Tetley and A. E. Porter, ACS Nano, 2017, 11, 2652-2664.

16 Y. Zhang, S. Tekobo, Y. Tu, Q. Zhou, X. Jin, S. A. Dergunov, E. Pinkhassik and B. Yan, ACS Appl. Mater. Interfaces, 2012, 4, 4099-4105.

17 L. Li, T. Liu, C. Fu, L. Tan, X. Meng and H. Liu, Nanomedicine, 2015, 11, 1915-1924.
18 J. Kim, S. V. Chankeshwara, F. Thielbeer, J. Jeong, K. Donaldson, M. Bradley and W. S. Cho, Nanotoxicology, 2016, 10, 94-101.

19 S. G. Elci, Y. Jiang, B. Yan, S. T. Kim, K. Saha, D. F. Moyano, G. Y. Tonga, L. C. Jackson, V. M. Rotello and R. W. Vachet, ACS Nano, 2016, 10, 5536-5542.

20 K. Saha, M. Rahimi, M. Yazdani, S. T. Kim, D. F. Moyano, S. Hou, R. Das, R. Mout, F. Rezaee, M. Mahmoudi and V. M. Rotello, ACS Nano, 2016, 10, 4421-4430.

21 T. E. Mikael, L. Martin, K. Egle, Š. Eimantas, B. G. Stefán and C. Tommy, Nanoscale Adv., 2019, 1, 1055-1061.

22 D. Magri, P. Sanchez-Moreno, G. Caputo, F. Gatto, M. Veronesi, G. Bardi, T. Catelani, D. Guarnieri, A. Athanassiou, P. P. Pompa and D. Fragouli, ACS Nano, 2018, 12, 7690-7700.

23 B. Gewert, M. M. Plassmann and M. MacLeod, Environ. Sci.: Processes Impacts, 2015, 17, 1513-1521.

24 O. S. Alimi, J. F. Budarz, L. M. Hernandez and N. Tufenkji, Environ. Sci. Technol., 2018, 52, 1704-1724.

25 K. Mattsson, L. A. Hansson and T. Cedervall, Environ. Sci.: Processes Impacts, 2015, 17, 1712-1721.

26 L. Nizzetto, M. Futter and S. Langaas, Environ. Sci. Technol., 2016, 50, 10777-10779.

27 J. M. Panko, J. Chu, M. L. Kreider and K. M. Unice, Atmos. Environ., 2013, 72, 192-199.

28 Y. Chae and Y. J. An, Mar. Pollut. Bull., 2017, 124, 624-632.

29 H. Bouwmeester, P. C. H. Hollman and R. J. B. Peters, Environ. Sci. Technol., 2015, 49, 8932-8947.

30 S. L. Wright and F. J. Kelly, Environ. Sci. Technol., 2017, 51, 6634-6647.

31 J. C. Prata, Environ. Pollut., 2018, 234, 115-126.

32 J. P. da Costa, P. S. M. Santos, A. C. Duarte and T. RochaSantos, Sci. Total Environ., 2016, 566, 15-26.

33 L. Wang, W. Wu, Bolan, S. Nanthi, Tsang, C. W. Daniel, Y. Li, M. Qin and D. Hou, J. Hazard. Mater., 2021, 401, 123415-123415.

34 R. Lehner, C. Weder, A. Petri-Fink and B. RothenRutishauser, Environ. Sci. Technol., 2019, 53, 1748-1765.

35 A. Bianco, F. Sordello, M. Ehn, D. Vione and M. Passananti, Sci. Total Environ., 2020, 742, 140413.

36 H. Zhang, Y. Y. Kuo, A. C. Gerecke and J. Wang, Sci. Total Environ., 2012, 46, 10990-10996.

37 B. Stephens, P. Azimi, Z. El Orch and T. Ramos, Atmos. Environ., 2013, 79, 334-339.

38 A. Batel, F. Linti, M. Scherer, L. Erdinger and T. Braunbeck, Environ. Toxicol. Chem., 2016, 35, 1656-1666.

39 K. Lindner, M. Stroebele, S. Schlick, S. Webering, A. Jenckel, J. Kopf, O. Danov, K. Sewald, C. Buj, O. Creutzenberg, T. Tillmann, G. Pohlmann, H. Ernst, C. Ziemann, G. Huettmann, H. Heine, H. Bockhorn, T. Hansen, P. Koenig and H. Fehrenbach, Part. Fibre Toxicol., 2017, 14, 8.

40 J. Liu, Y. Ma, D. Zhu, T. Xia, Y. Qi, Y. Yao, X. Guo, R. Ji and W. Chen, Environ. Sci. Technol., 2018, 52, 2677-2685.

41 I. Velzeboer, C. J. A. F. Kwadijk and A. A. Koelmans, Environ. Sci. Technol., 2014, 48, 4869-4876. 
42 R. Lehner, C. Weder, A. Petri-Fink and B. RothenRutishauser, Environ. Sci. Technol., 2019, 53, 1748-1765.

43 M. Shen, J. Xing, Q. Ji, Z. Li, Y. Wang, H. Zhao, Q. Wang, T. Wang, L. Yu, X. Zhang, Y. Sun, Z. Zhang, Y. Niu, H. Wang, W. Chen, Y. Dai, W. Su and H. Duan, Environ. Sci. Technol., 2018, 52, 6610-6616.

44 T. G. Iversen, T. Skotland and K. Sandvig, Nano Today, 2011, 6, 176-185.

45 H. Li, Y. Li, J. Jiao and H. M. Hu, Nat. Nanotechnol., 2011, 6, 645-650.

46 R. Firdessa, T. A. Oelschlaeger and H. Moll, Eur. J. Cell Biol., 2014, 93, 323-337.

47 V. Francia, K. Yang, S. Deville, C. Reker-Smit, I. Nelissen and A. Salvati, ACS Nano, 2019, 13, 11107-11121.

48 Q. Mu, G. Su, L. Li, B. O. Gilbertson, L. H. Yu, Q. Zhang, Y. P. Sun and B. Yan, ACS Appl. Mater. Interfaces, 2012, 4, 2259-2266.

49 R. Ali, S. Trump, I. Lehmann and T. Hanke, J. Biophotonics, 2015, 8, 361-371.

50 R. Barhoumi, Y. Mouneimne, E. Ramos, C. Morisseau, B. D. Hammock, S. Safe, A. R. Parrish and R. C. Burghardt, Toxicol. Appl. Pharmacol., 2011, 253, 45-56.

51 R. Barhoumi, J. M. Catania, A. R. Parrish, I. Awooda, E. Tiffany-Castiglioni, S. Safe and R. C. Burghardt, J. Toxicol. Sci., 2009, 34, 13-25.
52 E. T. W. Bampton, C. G. Goemans, D. Niranjan, N. Mizushima and A. M. Tolkovsky, Autophagy, 2005, 1, 2336.

53 H. J. Johnston, M. Semmler-Behnke, D. M. Brown, W. Kreyling, L. Tran and V. Stone, Toxicol. Appl. Pharmacol., 2010, 242, 66-78.

54 P. H. Hemmerich and A. H. von Mikecz, PLoS One, 2013, 8, 1-13.

55 T. Lammel, P. Boisseaux, M. L. Fernandez-Cruz and J. M. Navas, Part. Fibre Toxicol., 2013, 10, 1-21.

56 S. Munoz-Braceras, A. R. Tornero-Ecija, O. Vincen and R. Escalante, Dis. Models Mech., 2019, 12, 1-13.

57 A. Norambuena, H. Wallrabe, R. Cao, D. B. Wang, A. Silva, Z. Svindrych, A. Periasamy, S. Hu, R. E. Tanzi, D. Y. Kim and G. S. Bloom, EMBO J., 2018, 37, 1-18.

58 M. Qin, J. Zhang, M. Li, D. Yang, D. Liu, S. Song, J. Fu, H. Zhang, W. Dai, X. Wang, Y. Wang, B. He and Q. Zhang, Theranostics, 2020, 10, 1213-1229.

59 M. Iguchi, M. Hiroi, H. Kanegae and Y. Ohmori, Mediators Inflammation, 2018, 2018, 1-11.

60 R. F. Gahl;, P. Dwivedi and N. Tjandra, Cell Death Dis., 2016, 7, 1-11.

61 C. Rogers, D. A. Erkes, A. Nardone, A. E. Aplin, T. Fernandes-Alnemri and E. S. Alnemri, Nat. Commun., 2019, 10, 1-17. 